American Journal of Applied Sciences 8 (11): 1131-1134, 2011

ISSN 1546-9239

(C) 2011 Science Publications

\title{
Transient Response of a Novel Displacement Transducer for Magnetic Levitation System
}

\author{
${ }^{1}$ Mrunal Deshpande and ${ }^{2}$ Badrilal Mathur \\ ${ }^{1}$ EEE Department, SSN College of Engineering Rajiv Gandhi Salai, \\ (OMR), Kalavakkam 603110 Chennai, Tamilnadu India \\ ${ }^{2}$ EEE Depatment, SSN College of Engineering Chennai India
}

\begin{abstract}
Problem statement: In magnetic levitation system, position sensors are used to obtain a voltage proportional to the position of the suspended object. This is an essential feedback signal for stabilizing the system. These sensors make the system clumsy and prone to failures. To eliminate any physical attachment on the levitated object for the purpose of measuring its displacement, a novel magnetic displacement transducer has been designed. Approach: Variation in inductance of the transducer with the position of the levitated object was used to detect the position of the object. Coil of the transducer was excited by a $5 \mathrm{kHz}$ voltage and variation in phase angle of its current was measured by synchronous demodulation method. Transient response of this system was also obtained for step change in the position of the levitated object. Results: By simulation as well as by experiments it was observed that a minimum delay equal to one and a half times the cycle time of the exciting frequency was always present. The delay further increases with increase in order of the filter. In magnetic levitation applications, mechanical frequency of the levitated object was generally below $10 \mathrm{~Hz}$ and therefore a delay of around 300 micro seconds with an exciting frequency of $5 \mathrm{kHz}$ was acceptable. Steady state characteristic of the transducer was nearly linear and it was further linearized by using a look up table and cubic interpolation. Signal output from synchronous demodulation circuit had been digitally processed for application to magnetically levitated system. Conclusion: A novel yet simple circuit for sensing the position of the moving object for electromagnetic levitation system is developed. The transient response of the developed system is also obtained and the simulation results are verified experimentally.
\end{abstract}

Key words: Magnetic displacement transducer, magnetic levitation, current sensor, position sensors, transient response, signal output, cubic interpolation, synchronous demodulation, steady state, phase angle

\section{INTRODUCTION}

Magnetic levitation systems have practical importance in many Engineering applications such as high speed maglev trains, frictionless bearings etc. Of the various methods by which magnetic suspension can be achieved, the work here is concentrated on electromagnetic attraction type of magnetic levitation system. These kinds of systems are open-loop unstable. Therefore, feedback controllers (El Hajjaji and Ouladsine, 2001; Zhang and Suyama, 1995; Varatharaju et al., 2011) sliding mode control (AlMuthairi and Zribi, 2004; Deshpande and Mathur, 2011), neural (Lin et al., 2005) and fuzzy logic (Dukan et al., 2008) are used for stabilizing the position of the levitated object. Adaptive controllers commonly designed for motors (Husain et al., 2008) and Hybrid fuzzy controllers(Pratumsuwan et al., 2010) are also gaining popularity in control of servo systems.

An important signal required for the controller is the position of the levitated object. Optical sensors and Hall-effect sensors are generally used for detecting the position. These sensors are to be mounted on and near to the levitated object. This needs additional mountings that is either difficult or may be sometimes impossible. Earlier various novel sensing techniques have been designed by the authors.(Deshpande and Mathur, 2009; Deshpande and Badrilal, 2010). Yet another novel position sensor not using any moving part has been developed to overcome the drawbacks of conventional position sensors. Schematic of the proposed system is shown in Fig. 1.

Corresponding Author: Mrunal Deshpande, AP/EEE, SSN College of Engineering, Rajiv Gandhi Salai, (OMR), Kalavakkam 603110 Chennai, Tamilnadu India 
Am. J. Applied Sci., 8 (11): 1131-1134, 2011

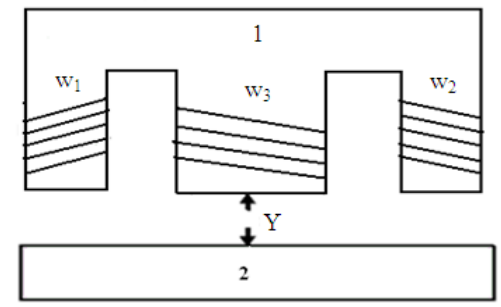

1-Core of lifting magnet, 2-Levitated object, w and $\mathrm{w}_{2}$-Lifting winding, $\mathrm{w}_{3}$-Displacement measuring winding, Y-Displacement

Fig. 1: Electromagnet and object to besuspended

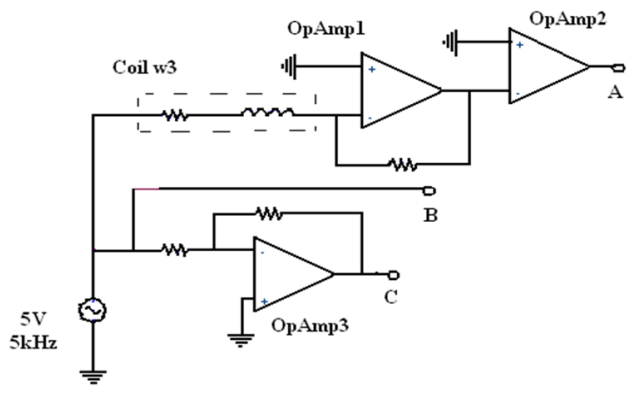

Fig. 2: Self sensing circuit

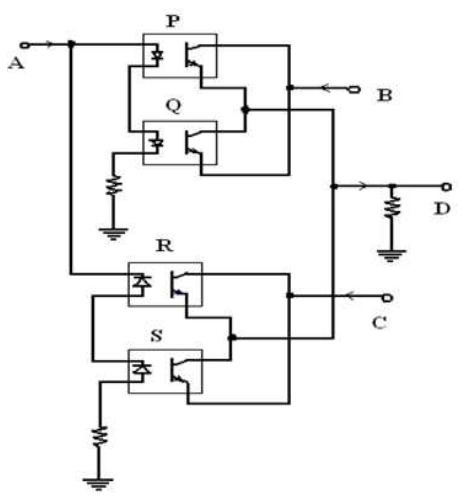

Fig. 3: Opto coupler multiplier

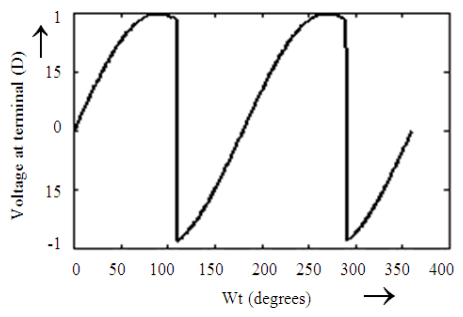

Fig. 4: Waveform of voltage at terminal $D$ versus $\omega t$
The electromagnet used is of $\mathrm{E}$ shape laminations and the levitated object is of I shape. Inductance of the lifting magnet is a function of the proximity of the levitated object and its inductance can be exploited for determination of the separation. The lifting coils $\mathrm{W}_{1}$ and $\mathrm{W}_{2}$ connected in series are located on the outer limbs of the core where as the distance measuring coil $\mathrm{W}_{3}$ is located on the central limb. The windings $\mathrm{W}_{1}$ and $\mathrm{W}_{2}$ are so connected that the flux produced by outer limbs is cancelled on the central limb.

The technique developed is based on the concept that variation in inductance of the central limb is a function of the position of the levitated object. The range of movement of the object is small as is the case in magnetic levitation. The variation in the inductance is observed as a phase displacement between current and voltage of the coil $\mathrm{W}_{3}$. The phase displacement is measured by the average value of a multiplier circuit. The average is determined by using a digital low pass filter. The simulation results show that a time delay of nearly one and half cycle of the exciting frequency of the coil $\mathrm{W}_{3}$ is introduced between displacement and signal output of the transducer. This delay is mainly due to the low pass filter. Simulation results were verified experimentally.

Self sensing circuit: In Fig. 2 the coil $\mathrm{W}_{3}$ is excited by $5 \mathrm{~V} 5 \mathrm{kHz}$. Impedance angle $\Phi+\alpha$ of the winding $\mathrm{W}_{3}$ is a function of the distance $\mathrm{Y} . \Phi$ is the phase difference when displacement $\mathrm{y}=0$.

Opamp1 forms a current sensor which converts the coil current into a proportional voltage without introducing any impedance in the measuring circuit. Opamp2 converts this voltage into square wave at point $\mathrm{A}$. Phase angle of the square wave with respect to the applied voltage is $(\Phi+\alpha)$ where $\alpha$ is the phase difference introduced due to displacement $\mathrm{Y}$. A voltage proportional to the applied voltage to $\mathrm{W}_{3}$ is derived directly at point $\mathrm{B}$ and its inverted value through unity gain inverting Opamp3 at point $\mathrm{C}$.

Opto coupler multiplier: Voltages at points A, B and $\mathrm{C}$ of Fig. 2 are multiplied by an opto-coupler multiplier shown in Fig. 3. LEDs of opto-couplers $\mathrm{P}$ and $\mathrm{Q}$ are connected in series. LEDs of $\mathrm{P}$ and $\mathrm{Q}$ are $\mathrm{ON}$ when the voltage of point $A$ is positive. Similarly LEDs of $R$ and $\mathrm{S}$ are $\mathrm{ON}$ when $\mathrm{A}$ is negative. Collectors and emitters of BJTs of $\mathrm{P}$ and $\mathrm{Q}$ are connected in anti parallel and similarly, collectors and emitters of BJTs of R and S are also connected in anti parallel. A conducting path is established between $\mathrm{B}$ and $\mathrm{D}$ when $\mathrm{A}$ is positive and between $\mathrm{C}$ and $\mathrm{D}$ when $\mathrm{A}$ is negative. Wave shape of the voltage at $\mathrm{D}$ is shown in Fig. 4. Proportions of the 
positive and negative areas depend on the phase difference between sinusoidal voltage at points B and C and square wave voltage at point $\mathrm{A}$.

\section{MATERIALS AND METHOD}

Method of measurement of distance $y$ and inductance: The distance $\mathrm{y}$ was measured by a travelling microscope and the inductance of the coil w3 was measured by an auto balancing a.c. bridge.

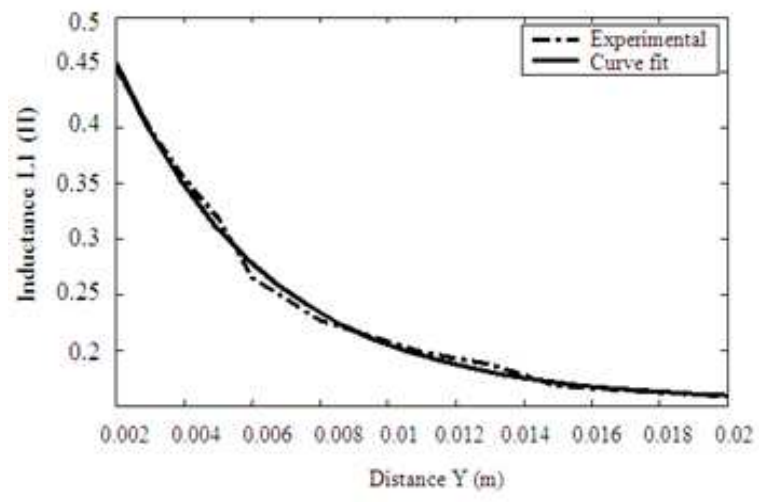

Fig. 5: Distance Y versus inductance L1

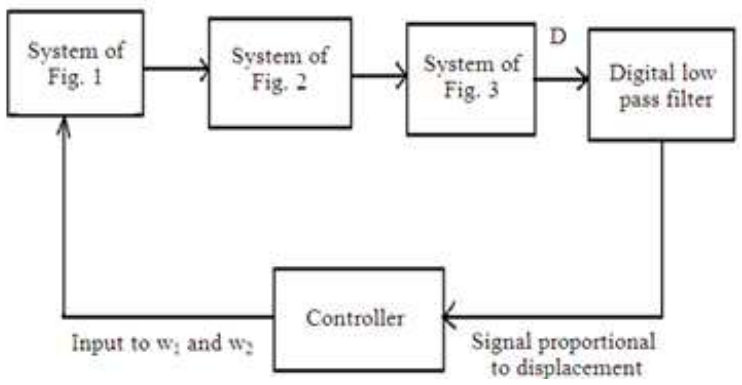

Fig. 6: Overall block diagram of the system

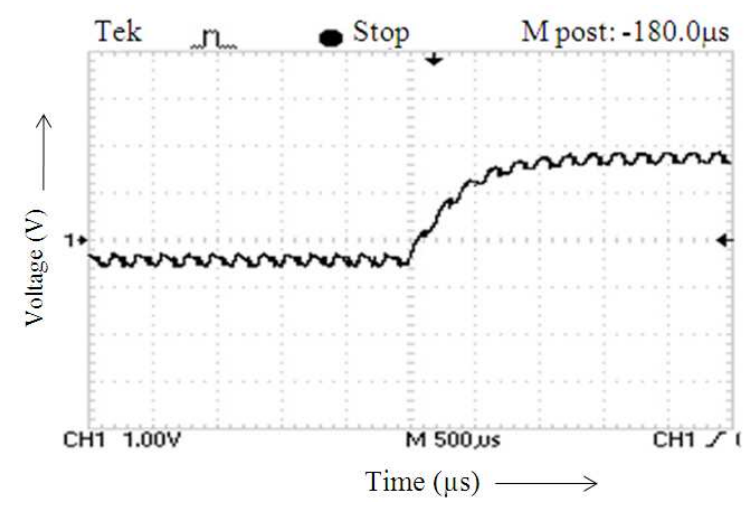

Fig. 7: Transient response of the proposed transducer
Table: 1 Materials Used

\begin{tabular}{llll}
\hline S No & Item & Refer Fig. & Material \\
\hline 1 & $\begin{array}{l}\text { Core of lifting magnet } \\
\text { and Levitated object }\end{array}$ & Fig. 1 & Silicon Steel \\
& & Laminations \\
2 & Opamp1,2 and 3 & Fig. 2 & LM741 \\
3 & Optocoulers P,Q,R,S & Fig. 3 & 4N25 \\
4 & $\begin{array}{l}\text { Digital Low pass filter and } \\
\text { controller }\end{array}$ & Fig. 6 & TMS320LF2407 \\
\hline
\end{tabular}

The inductance versus y plot obtained experimentally is shown in Fig. 5. To smooth out the experimental readings an exponential curve fitting was done. The relation between displacement and inductance is expressed as:

$$
\mathrm{L}_{1}=\mathrm{a}^{*} \exp \left(\mathrm{b}^{*} \mathrm{y}\right)+\mathrm{c}^{*} \exp \left(\mathrm{d}^{*} \mathrm{y}\right)
$$

where, $\mathrm{a}=0.4737, \mathrm{~b}=-227.2157, \mathrm{c}=0.1578$ and $\mathrm{d}=-0.9830$

\section{RESULTS}

Simulation and experimental results: Systems shown in Fig. 1-3 were connected in cascade as shown in Fig. 6. Output D of Fig. 3 was given to a Micro Controller. The microcontroller converts the analog voltage at point $\mathrm{D}$ into digital and applies it to a two stage IIR low pass filter having a cut off frequency of $100 \mathrm{~Hz}$.

Steady state response: Output of the low pass filter is a DC voltage which is proportional to $\sin (\Phi+\alpha)$. Relation between the filter output $\mathrm{V}$ and the displacement $\mathrm{Y}$ was found to be nearly linear.

To further improve the linearity, the digital signal output from the low pass filter was applied to a look-up Table. 1 and cubic interpolation was used to improve the linearity.

Transient response of the proposed transducer: A step change was made in the displacement $\mathrm{Y}$ at 500 microsecond and corresponding change in $\mathrm{V}$ was recorded Fig. 7 using digital storage oscilloscope, Agilent 7000B.The voltage $\mathrm{V}$ took a time of about 300 microseconds to change from one steady state value to the other. This time delay is one and half times the cycle time of the exciting frequency of $5 \mathrm{KHz}$ for the coil W3. The delay also depends upon the order of the filter and its cut off frequency. The results obtained experimentally and also by simulation are matching. 


\section{DISCUSSION}

As the lifting coils w1 and w2 should be magnetically decoupled from the measuring coil w3 of Fig. 1, the choice of core shapes are limited. Exciting frequency of the coil w3 should be very high as compared to the mechanical frequency of oscillations of the levitated object. For every high frequency of excitation, ferrite may be used for lifting and levitated objects of Fig. 1.

\section{CONCLUSION}

A novel yet simple circuit for sensing the position of the moving object for electromagnetic levitation system is developed. A linear relationship is obtained between the voltage and the position. The variation in phase of output voltage with respect to inductance and hence the position of the moving object is obtained. The transient response of the developed system is also obtained and the simulation results are verified experimentally. Simplicity of the designed circuit and absence of moving parts make it more attractive for magnetic levitation applications.

\section{REFERENCES}

Dukan, A.V., M. Abrudean and M. Dulau, 2008. Positioning system based on electromagnetic levitation using fuzzy learning control. CEAI, 10: 60-69.

Al-Muthairi, N.F. and M. Zribi, 2004. Sliding Mode Control of a Magnetic Levitation System. Mathem. Problems Eng., 2: 93-107.

El Hajjaji, A. and M. Ouladsine, 2001. Modeling and nonlinear control of magnetic levitation systems. IEEE, Trans. Indus. Electronics, 48: 831-838. DOI: $10.1109 / 41.937416$

Lin, F.J., H.J. Shieh, L.T. Teng and P.H. Shieh, 2005. Hybrid controller with recurrent neural network for magnetic levitation system. IEEE Trans. Magnetics, 41: 2260-2269. DOI: 10.1109/TMAG.2005.848320
Husain, H., M. Khalid and R. Yusof, 2008. Direct model reference adaptive controller based-on neural-fuzzy techniques for nonlinear dynamical systems. Am. J. Applied Sci., 5: 769-776. DOI: 10.3844/ajassp.2008.769.776

Deshpande, M. and B.L. Mathur, 2009. A novel displacement sensor for magnetic levitation, Int. J. Recent Trends Eng., 1: 21-24.

Deshpande, M. and B.L. Mathur, 2011. Auto sensing Controller for Magnetic Levitation System. Eur. J. Sci. Res., 49: 200-207.

Deshpande, M. and M. Badrilal, 2010. Sensorless control of magnetic levitation system using sliding mode control. Proceedings of the International Conference on Computer Applications and Industrial Electronics, Dec. 5-8, IEEE Xplore Press, Kuala Lumpur, pp: 9-14. DOI: 10.1109/ICCAIE.2010.5735037

Pratumsuwan, P., S. Thongchai and S. Tansriwong, 2010. A hybrid of fuzzy and proportional-integralderivative controller for electro-hydraulic position servo system. Energy Res. J., 1: 62-67. DOI: $10.3844 /$ erjsp.2010.62.67

Varatharaju, V. M., B. Mathur and Udhayakumar, 2011. Adaptive Controllers for Permanent Magnet Brushless DC Motor Drive System using AdaptiveNetwork-based Fuzzy Interference System. Am. J. Applied Sci., 8: 810-815. DOI: 10.3844/ajassp.2011.810.815

Zhang, F. and K. Suyama, 1995. Nonlinear feedback control of magnetic levitating system by exact linearization approach. Proceedings of the 4th IEEE Conference on Control Applications, Sep. 28-29, IEEE Xplore Prss, Albany, NY., USA., pp: 267-268. DOI: 10.1109/CCA.1995.555713 